\title{
O uso da simulação no treinamento da gestão em saúde
}

\author{
Using of simulation in healthcare management training
}

El uso de la simulación en la formación en gestión en salud

\begin{abstract}
Gabriela Furst Vaccarezza¹, Dario Cecilio-Fernandes², Carolina Felipe Soares Brandão.
\end{abstract}
\section{RESUMO}

Objetivo: Descrever a inserção curricular e analisar a satisfação dos discentes em medicina sobre o ensino de gestão baseado em simulação, em particular no manejo de maus tratos a criança. $O$ ensino de gestão, geralmente ocorre de forma teórica, e no ambiente real muitas vezes não é possível ou homogêneo abordar os todos os casos necessários. Métodos: Estudo exploratório de abordagem quantitativa em questionário aplicado a 57 discentes do oitavo semestre de medicina. Os dados foram analisados com estatísticas descritivas e o estudo foi aprovado por Comitê de Ética em Pesquisa. Resultados: Todos os discentes consideraram estas simulações fundamentais para a formação e a sugerem como temática obrigatória no curso. A percepção sobre o foco da atividade teve grande variabilidade, tanto no foco percebido como no desejado pelos estudantes, uma vez que a assistência técnica é supervalorizada, mesmo em cenários onde a condução assistencial e administrativa possa significar o bem-estar do paciente. Conclusão: Não há relatos publicados sobre implementação de simulação na área de gestão na graduação, logo o estudo evidencia sua importância para que possa ser vivenciado e compartilhado emoções e condutas em ambiente controlado para atuação médica de qualidade.

Palavras-chave: Educação em saúde, Simulação, Gestor de saúde, Estudantes de medicina, Prática de saúde pública.

\begin{abstract}
Objective: To describe the insertion and analyze medical students' satisfaction with teaching health management using simulation training, particularly in the management of child maltreatment. Teaching health management often focuses on the theoretical aspects, since the practical aspects are often impossible to address in primary care. Methods: Exploratory study of quantitative approach in a questionnaire applied to 57 students of the eighth semester of medicine. Data were analyzed with descriptive statistics. This study was approved by the University Research Ethics Committee. Results: All students considered simulations fundamental to their training and suggested them as a mandatory subject in the course. The perception about the focus of the activity had great variability, both in the students' perceived and desired focus, since technical assistance is overvalued, even in scenarios where care and administrative management may mean patient well-being. Conclusion: There are no published research on the implementation of simulation in the area of teaching in health management for undergraduate students. This study highlights the importance of simulation, especially on emotions and behaviors that can be experienced and shared in a controlled environment for quality medical practice.
\end{abstract}

Keywords: Health education, Simulation techique, Health manager students medical, Public health practice.

\footnotetext{
${ }^{1}$ Universidade Municipal de São Caetano (USCS), São Caetano do Sul - SP.

*E-mail: carolinafs11@gmail.com

2 Universidade Estadual de Campinas (UNICAMP), Campinas - SP.
}

SUBMETIDO EM: 1/2022

ACEITO EM: 2/2022

PUBLICADO EM: 2/2022 


\section{RESUMEN}

Objetivo: Describir la inserción curricular y analizar la satisfacción de los estudiantes de medicina sobre la enseñanza de la gestión basada en la simulación, en particular en el manejo de los malos tratos a los niños. La enseñanza de la gestión, por lo general, se desarrolla de forma teórica, y en el entorno real muchas veces no es posible u homogéneo abordar todos los casos necesarios. Métodos: Estudio exploratorio de enfoque cuantitativo en cuestionario aplicado a 57 estudiantes del octavo semestre de medicina. Los datos se analizaron con estadísticas descriptivas y el estudio fue aprobado por el Comité de Ética de la Investigación. Resultados: Todos los ponentes consideraron estas simulaciones fundamentales para la formación y sugirieron la temática necesaria en el curso. La percepción sobre el foco de la actividad tuvo una gran variabilidad, tanto en el foco percibido como en el deseado por los estudiantes, una vez que la asistencia técnica es muy valorada, incluso en cenários donde la conducción asistencial y administrativa puede significar el bienestar del paciente. Conclusión: No hay relatos publicados sobre la implementación de la simulación en el área de la gestión en la gradación, pero el estudio evidencia su importancia para que puedan vivirse y compartirse emociones y conductas en un ambiente controlado para la actuación médica de calidad.

Palabras clave: Educación en salud, Simulación, Gestión de salud, Estudiantes de medicina, Práctica de salud pública.

\section{INTRODUÇÃO}

Nos últimos vinte anos, o ensino médico no Brasil foi foco de diversos debates, com ênfase na implantação de reformas curriculares para os cursos de Medicina. Estas reformas, são guiadas pelas Diretrizes Curriculares Nacionais (DCN), que substituíram o rigor de formas e conteúdo das grades do currículo mínimo, possibilitando a inovação e a diversificação na formação do profissional por meio da flexibilização da construção de projetos pedagógicos mais condizentes com o pensamento contemporâneo. Tais mudanças visaram aproximar o médico às necessidades sociais e de saúde da população brasileira (MINISTÉRIO DA EDUCAÇÃO, 2014; KUSSAKAWA DHB e ANTONIO CA, 2017).

A DCN preconiza que o médico brasileiro tenha uma formação generalista, humanista com competência para atuar nos diferentes níveis de atenção à saúde. Sempre com compromisso ético e com responsabilidade social por meio de ações de reabilitação, prevenção e promoção à saúde. Este egresso deve ser capaz de atuar para responder as necessidades em saúde individual e coletiva da população. $O$ perfil médico brasileiro na graduação preconiza uma formação de generalista. Tal formação é constituída de forma humanista, crítica, reflexiva e ética. Além disso, o médico generalista tem que ter a capacidade de atuar nos diferentes níveis de atenção à saúde, com ações de promoção, prevenção, recuperação e reabilitação da saúde, tanto no âmbito individual quanto coletivo. Ademais, precisa atuar com responsabilidade social e compromisso com a defesa da cidadania e da dignidade humana, entendendo 0 ser humano de forma integral (MINISTÉRIO DA EDUCAÇÃO, 2014).

Em particular, destaca-se os desafios de formar um profissional com competência em gestão em saúde que visa um médico com capaz de promover o bem-estar da comunidade, por meio de ações que respondam as necessidades em saúde coletiva e necessidade em saúde individual seguindo as políticas e princípios do SUS (MINISTÉRIO DA EDUCAÇÃO, 2014).

A prática da medicina e os ambientes organizacionais, em que ocorre, tem crescido em complexidade ao longo tempo. A prática médica está repleta de desafios da gestão e liderança, contudo as habilidades de gestão são frequentemente subdesenvolvidas e muitas vezes subestimadas, na área da saúde. Sendo assim, a transformação da gestão do trabalho em saúde envolve diversas questões, que não estão restritas as técnicas, mas também que envolvem mudanças para dentro e para fora das instituições de ensino (MYERS CG e PROVONOST PJ, 2017).

A gestão em saúde não é simplesmente uma forma de organizar os serviços de saúde, nem um modo de administrar um sistema de saúde, mas reflete uma combinação de saberes e técnicas que são utilizados 
para resolver problemas e atender a necessidades de saúde, individuais e coletivas (MINISTÉRIO DA EDUCAÇÃO, 2015). Dessa forma, o desenvolvimento das competências dessa área envolve um ambiente com alto grau de disputas entre heterogêneos atores sociais, onde decisões e atuações são arroladas levando em conta diferentes interesses sociais, políticos e técnico-assistenciais (BURSZTYN I, 2015).

A gestão em saúde pública é de grande complexidade, pois integra todos os níveis de complexidades do Sistema Único de Saúde (SUS), que visa a cobertura universal e gratuita de saúde no território brasileiro. Além dos diferentes níveis de complexidade, variando da primárias mais simples a terciária maior complexidade, o SUS também é compartilhado pelo governo municipal, estadual e federal. Considera se, portanto, que a gestão em saúde é normalmente resultante das influências e governabilidade em que diferentes atores sociais são capazes de exercer em cenário e tempo determinados. Os desafios do ensino em gestão em saúde são vários, variando desde o local de prática até questões organizacionais entendendo o limite de atuação nas diferentes esferas de governo (BURSZTYN I, 2015).

Ainda, existe o desafio de que a gestão em saúde está ligada a diferentes cargos e estruturas de poder, dificultando assim a integração ensino-serviço em todos os níveis de atenção. Isso, em especial, vem criando grandes dificuldades para o ensino da gestão em saúde, além de sua parte teórica. A simulação em saúde vem sendo utilizado para ensinar componentes práticos importantes para a atuação profissional em que muitas vezes não é possível no ensino de forma plena, muitas vezes por ser um evento isolado, raro ou ainda que frequente não ser necessariamente presenciado por todos os estudantes de forma simultânea. A simulação em saúde é considerada uma metodologia de ensino na qual visa replicar o ambiente de real de prática para o ensino de habilidades e competências (GABA DM, 2007; MCGAGHIE WC, et al., 2010; BRANDÃO CFS e FERNANDES DC, 2018; BRANDÃO CS, et al., 2018).

A simulação também permite o ensino em um ambiente seguro para o aluno, possibilitando que o mesmo possa aprender com o erro, e o paciente, pois prepara o aluno para a prática assim como não o coloca em risco (GABA DM, 2007; BRANDÃO CFS e FERNANDES DC, 2018; BRANDÃO CS, et al., 2018). $O$ ensino baseado em simulação pode usar diversas tecnologias como manequins, softwares, robôs ou atores com um objetivo pré-determinado (COOK DV, et al., 2011; MOTOLA I, et al., 2013). Finalmente, a simulação vem sendo cada vez mais incluído no ensino de graduação (BRANDÃO CS, et al., 2018).

Neste contexto, podemos dizer que a simulação é um ambiente controlado onde as temáticas podem ser vivenciadas e discutidas de forma uniforme, um benefício em relação ao ambiente real, que não há como ser controlado e totalmente uniformizado. A simulação é uma metodologia que contempla diversas técnicas, a depender dos objetivos de aprendizagem, público-alvo e até mesmo orçamento disponível. Neste contexto, há simuladores extremamente realísticos, manequins de baixo custo para treino de habilidades, utilizações de atores e possibilidades mediadas por tecnologias (BRANDÃO CS, et al., 2018).

A capacitação dos estudantes de medicina, na gestão em saúde, tem foco nas melhores práticas em situações de gestão de pessoas e prática organizacional geral, além de promover atualizações, reflexões e análises em situações de conflito e reforçar as diretrizes e as políticas públicas vigentes (BURSZTYN I, 2015).

Treinar estes futuros profissionais a uma formação efetiva pelo tamanho e complexidade que o SUS apresenta, e até mesmo pela falta de literatura nesta temática em si para sua inclusão curricular, foi estruturado dentro do módulo de simulação que ocorre simultaneamente ao estágio na atenção primária em saúde, temáticas com foco na gestão em saúde. O objetivo deste estudo foi avaliar a percepção dos discentes na atividade simulada de gestão em saúde assim como a sua inserção curricular. Descrever a percepção discente desta implementação e quais temáticas foram consideradas relevantes ao currículo brasileiro.

\section{MÉTODOS}

Estudo exploratório de abordagem quantitativa, a partir das respostas dos discentes do Curso de Medicina em um questionário fechado após exposição a atividade simulada com objetivo na capacitação em gestão em saúde. 


\section{Inserção curricular}

O objetivo primário da simulação foi manejar e reconhecer situação de risco de uma criança. Neste contexto é necessário conhecer do ponto de vista administrativo o sistema de saúde, para melhor atender os pacientes e familiares envolvidos. Para o desenvolvimento da simulação de Gestão em Saúde, os coordenadores dos serviços de simulação, dos serviços da comunidade e a coordenação do curso realizaram reuniões para pontuar os casos mais relevantes e prevalentes, as técnicas em simulação mais adequadas e custos para o desenvolvimento destas atividades.

A decisão da equipe docente e gestão do curso de medicina foi incluir estas atividades durante o oitavo semestre. Nesta fase os alunos já reconheceram e participaram ativamente do sistema público, seja em unidades básicas de saúde ou ambulatórios, com intensa percepção sobre o matriciamento da rede, que é um processo de construção compartilhada entre pelo menos duas equipes de saúde criando um plano terapêutico singular para o paciente (CHIAVERINI DH, et al., 2011).

Neste momento, os alunos também já tiveram o conteúdo necessário tanto da exposição do ambiente real do sistema público brasileiro como da exposição a diferentes técnicas de simulação incluídas de forma longitudinal no curso. A simulação em gestão em saúde foi incluída dentro do próprio módulo de simulação, que nesta etapa aborda temáticas com foco em urgências e emergências clínicas. Esse módulo possui em seu cronograma simulações focadas na questão técnica, com cenários que exigem raciocínio clínico e condutas procedimentais com simuladores de alta fidelidade nos pacientes adulto, pediátrico e obstétrico.

Estas simulações são programadas por um período de 4 semanas, totalizando 16 horas por grupo ao longo do semestre. Este módulo ocorre sempre no período da tarde, para grupos de no máximo 12 alunos, sendo que no outro período há rodízio de turmas com o ambiente de atendimento real em unidade básica de saúde. Esta decisão refere se a geralmente o atendimento nos ambientes não controlados serem mais intensos no período da manhã e desta forma minimizar impactos na aprendizagem destes estudantes no ambiente real. Após estas 4 semanas, este grupo de estudantes partem para um outro rodízio para vivenciar outras experiências em ambientes não controlados em diferentes especialidades.

Esta inclusão da simulação em gestão em saúde foi realizada no primeiro semestre de 2021, seguindo todos os protocolos institucionais estipulados para atividades práticas no período de pandemia pelo COVID19. A reorganização das temáticas fez com que as 4 horas do total em simulação fossem dedicados a cenários que envolvessem a necessidade de conhecimentos em gestão.

Foram realizados quatro cenários de diferentes esferas que foram discutidos também pela realidade local a qual estes estudantes estão inseridos na comunidade: maus tratos na infância, comunicação de sífilis para gestante, absenteísmo da equipe de trabalho e atestado de óbito.

\section{Simulação em Gestão e Administração em Saúde}

A temática escolhida para exemplificar estas atividades baseadas em simulação clínica foi o de maus tratos na infância, descrito no anexo desta pesquisa A Organização Mundial da Saúde (OMS) e o Ministério de Saúde Brasileiro colocam as crianças e adolescentes como as principais vítimas de violência, principalmente da violência intrafamiliar (RODRIGUES NCP, et al., 2017; FREITAS RJM, et al., 2016).

O objetivo desta atividade pode ser considerado como misto, uma vez que é fundamental o reconhecimento e manejo inicial técnico deste paciente, assim como uma efetiva comunicação com o familiar e seguimento administrativo sobre notificação de acordo com a legislação brasileira. As técnicas escolhidas para atingir os objetivos de aprendizagem foram a simulação seguida de debriefing, conhecida como simulação padrão, com o uso de um simulador pediátrico de alta fidelidade como paciente a ser examinado e um ator ou atriz que realizará a função do responsável pela criança para atingir os objetivos comportamentais.

O robô e atores podem ser utilizados associados ou somente com o paciente robô com as informações do familiar repassadas pelo facilitador/docente $O$ debriefing, foi conduzido por 2 docentes, sendo um deles com expertise na parte técnica do atendimento e por outro docente especialista em gestão em saúde. Os 
dois docentes são capacitados na técnica da simulação envolvida de modo a considerar a proteção psicológica dos participantes, pelo teor da atividade, que certamente desencadea sensações negativas que devem ser trabalhadas adequadamente.

Entretanto, independente das temáticas simuladas escolhidas, os quatro objetivos da gestão e administração em saúde, foram selecionados baseados com as indicações das Diretrizes Nacionais Curriculares Brasileiras que estão vigentes desde 2014 (MINISTÉRIO DA EDUCAÇÃO, 2014). Tais temas estão descritos na Figura 1.

Figura 1 - Fluxograma dos quatro objetivos da simulação com foco em gestão em saúde.

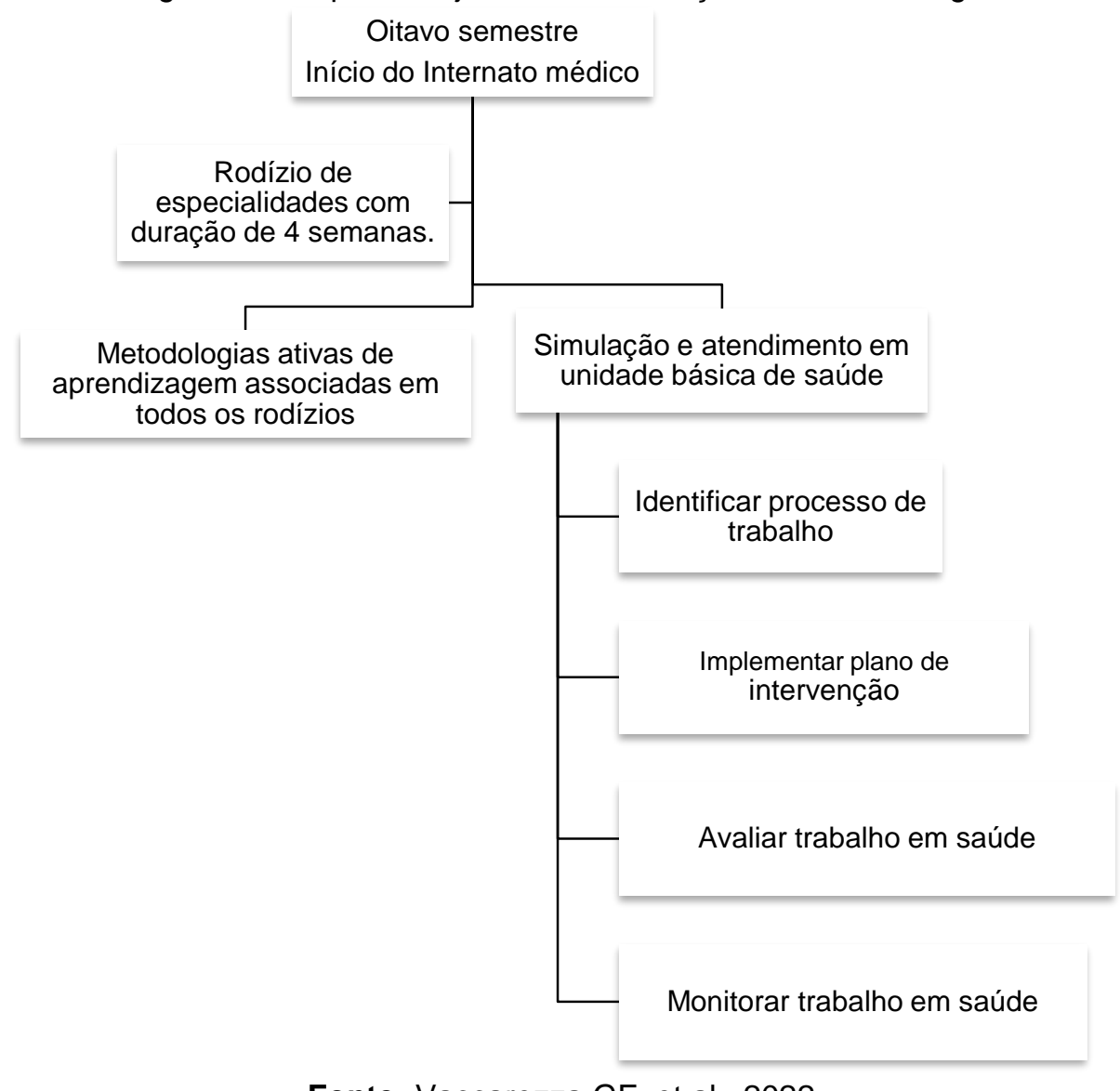

Fonte: Vaccarezza GF, et al., 2022.

As temáticas discutidas baseadas em simulação clínica foram também atreladas as Diretirzes Curriculares Brasileiras, destacando um objetivo central conforme Quadro 1.

Quadro 1 - Temáticas das atividades baseadas em simulação com foco na gestão em saúde.

\begin{tabular}{|c|c|c|}
\hline DCNs & Temática e Metodologia & Objetivo Central \\
\hline $\begin{array}{c}\text { Identificação do processo } \\
\text { de trabalho }\end{array}$ & $\begin{array}{c}\text { Gestante com sífilis - Paciente } \\
\text { Estandarizado }\end{array}$ & $\begin{array}{c}\text { Notificação compulsória e fluxo de } \\
\text { comunicação com o paciente / } \\
\text { contactantes. }\end{array}$ \\
\hline $\begin{array}{c}\text { Monitoramento do } \\
\text { trabalho em saúde }\end{array}$ & $\begin{array}{c}\text { Atestado de óbito - Paciente } \\
\text { Estandarizado }\end{array}$ & Manejo da morte encefálica \\
\hline $\begin{array}{c}\text { Implementação de plano } \\
\text { de intervenção }\end{array}$ & $\begin{array}{c}\text { Maus tratos - Paciente } \\
\text { Estandarizado com atendimento ao } \\
\text { simulador pediátrico }\end{array}$ & $\begin{array}{c}\text { Identificação da clínica. Conselho } \\
\text { tutelar - Vara da infância e } \\
\text { juventude. }\end{array}$ \\
\hline $\begin{array}{c}\text { Avaliação do trabalho em } \\
\text { saúde }\end{array}$ & $\begin{array}{c}\text { Avaliação de indicadores/individual } \\
\text { (absenteísmo) - paciente } \\
\text { Estandarizado }\end{array}$ & $\begin{array}{c}\text { Avaliação de indicadores e feedback } \\
\text { institucional. }\end{array}$ \\
\hline
\end{tabular}

Fonte: Vaccarezza GF, et al., 2022. 


\section{Instrumento}

O instrumento elaborado pelos docentes foi dividido em três blocos A primeira parte foi relacionado a dados demográficos, como gênero, idade, outra formação acadêmica e, se tinham algum conhecimento sobre as DCN. Além disso, foi perguntado (1) se acreditavam que a simulação deveria ser uma atividade obrigatória, e (2) se essa discussão em si seriam cenários importantes neste momento do desenvolvimento na graduação.

O segundo bloco, foram perguntas relacionadas a visão de prioridade do foco da atividade. Subsequentemente, os alunos colocaram em ordem decrescente qual seria o objetivo mais relevante. Como terceiro e último bloco, os estudantes foram convidados a colocar os sentimentos que a atividade despertou, e neste caso foi aceito mais de uma resposta. Também neste bloco foi perguntado se eles se sentiam mais capacitados para atender este tipo de situação simulada e se já haviam presenciado nos ambientes não controlados de aprendizagem este tipo de conflito.

\section{Coleta de dados e análise de dados}

Todos os estudantes foram convidados a responder as questões logo após o debriefing e após aceite do termo de conhecimento livre esclarecido. Não foi fornecido nenhuma instrução na maneira em como responder as questões, e o fizeram em uma sala apropriada e separada dos docentes que realizaram a atividade a fim de evitar qualquer constrangimento durante o preenchimento. Os dados foram analisados com estatísticas descritivas. A pesquisa teve o aceite da Comissão de Ética em Pesquisa, registrada sob o número 52071921.9.0000.5510, e parecer número 5.023.466.

\section{RESULTADOS}

O questionário foi respondido imediatamente após a atividade por 57 alunos, o que representa $95 \%$ dos alunos matriculados neste período do curso. Destes, $60 \%$ (34) eram do gênero feminino e $40 \%$ (23) do gênero masculino.

A idade dos estudantes variou de 21 a 30 anos com média de 23 anos. Ainda, dois alunos tiveram formação prévia (dentista e engenheiro); quatro alunos relataram já terem vivenciado na prática cenário similar (maus tratos); dois alunos relataram não sentir se mais preparados para atenderem casos de maus tratos, pois precisam amadurecer emocionalmente, uma vez que há abuso infantil associado. Todos os discentes $(100 \%)$ consideraram a simulação fundamental em sua formação e acreditam que a simulação com esta temática deva ser obrigatória. Quanto ao sentimento que a atividade despertou obtivemos 89 respostas com nove sentimentos diferentes. Tristeza foi presente em quase metade dos sentimentos relatados $(47 \%)$, seguido por raiva $(28 \%)$, surpresa $(7 \%)$, indignação $(6 \%)$, impotência $(4 \%)$, medo $(3 \%)$, angústia (2\%), empatia e preocupação (1\%).

A percepção discente do foco da atividade teve grande variabilidade, tanto no foco percebido como no foco desejado pelos estudantes. O maior consenso foi que o exame físico do paciente pediátrico foi o foco mais importante da atividade (44\%). A ordem decrescente do foco do cenário compreendido pelos estudantes foi: 1- Exame físico do paciente pediátrico (44\%), 2- Exame físico do paciente pediátrico (32\%) e emergência em pediatria (23\%), 3- Anamnese dirigida (26\%), 4- uso de exames complementares (21\%), 5- Notificação de agravos (19\%), 6- uso de exames complementares e gestão em saúde (23\%), 7Indicadores de saúde (35\%) e 8- Indicadores de saúde (35\%) e gestão em saúde (30\%).

No Gráfico 1 é possível observar todas as respostas de qual foi o objetivo da atividade percebido pelos discentes em ordem decrescente. Como foco principal da atividade os resultados foram: Emergência em pediatria $(32 \%)$, exame físico do paciente pediátrico (44\%), Notificação de agravos (11\%), Anamnese dirigida (7\%), Habilidades em comunicação $(9 \%)$, Uso de exames complementares (2\%). Como menor foco percebido foi: Indicadores de saúde (35\%), Gestão em saúde (30\%), Uso de exames complementares (21\%), Anamnese dirigida (4\%), Habilidade de comunicação (4\%) Notificação de agravo (2\%). 
Gráfico 1 - Percepção discente sobre o objetivo do cenário de maus tratos descritos pelos docentes.

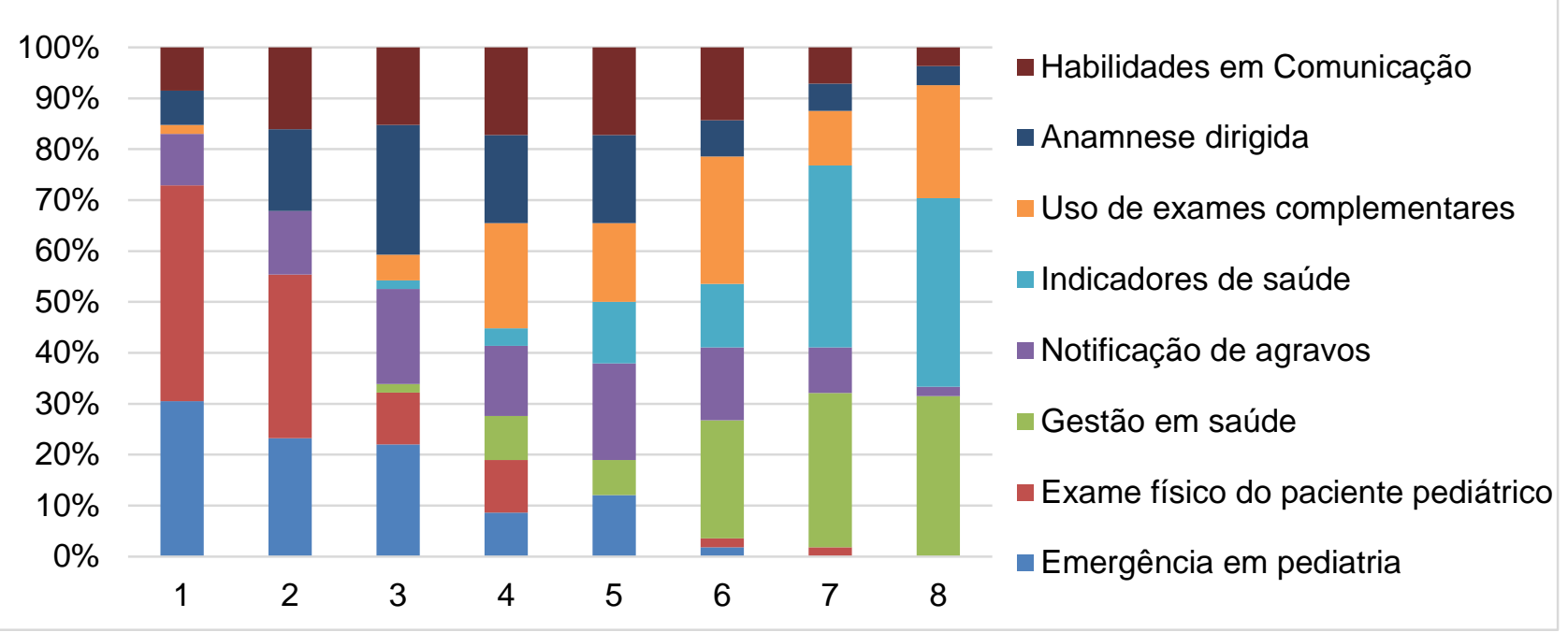

Fonte: Vaccarezza GF, et al., 2022.

Quanto a percepção discente de qual deveria ter sido o objetivo principal do cenário de maus tratos, sete dos oito itens possíveis foram assinalados. Emergência em pediatria (35\%), Exame físico do paciente pediátrico (28\%), Gestão em saúde (4\%), Notificação de agravos (5\%), indicadores de saúde (4\%), anamnese dirigida (18\%), Habilidades em comunicação (12\%). A sequência em ordem decrescente desejável segundo os estudantes seria: Emergência em pediatria (35\%), Anamense Dirigida (26\%) e Emergência em pediatria (26\%), Exame físico do paciente pediátrico (28\%), uso de exames complementares (25\%), habilidades em comunicação (28\%), Indicadores de saúde (44\%), gestão em saúde (37\%) conforme demonstrado no Gráfico 2.

Gráfico 2 - Percepção discente qual deveria ser o objetivo do cenário de maus tratos.

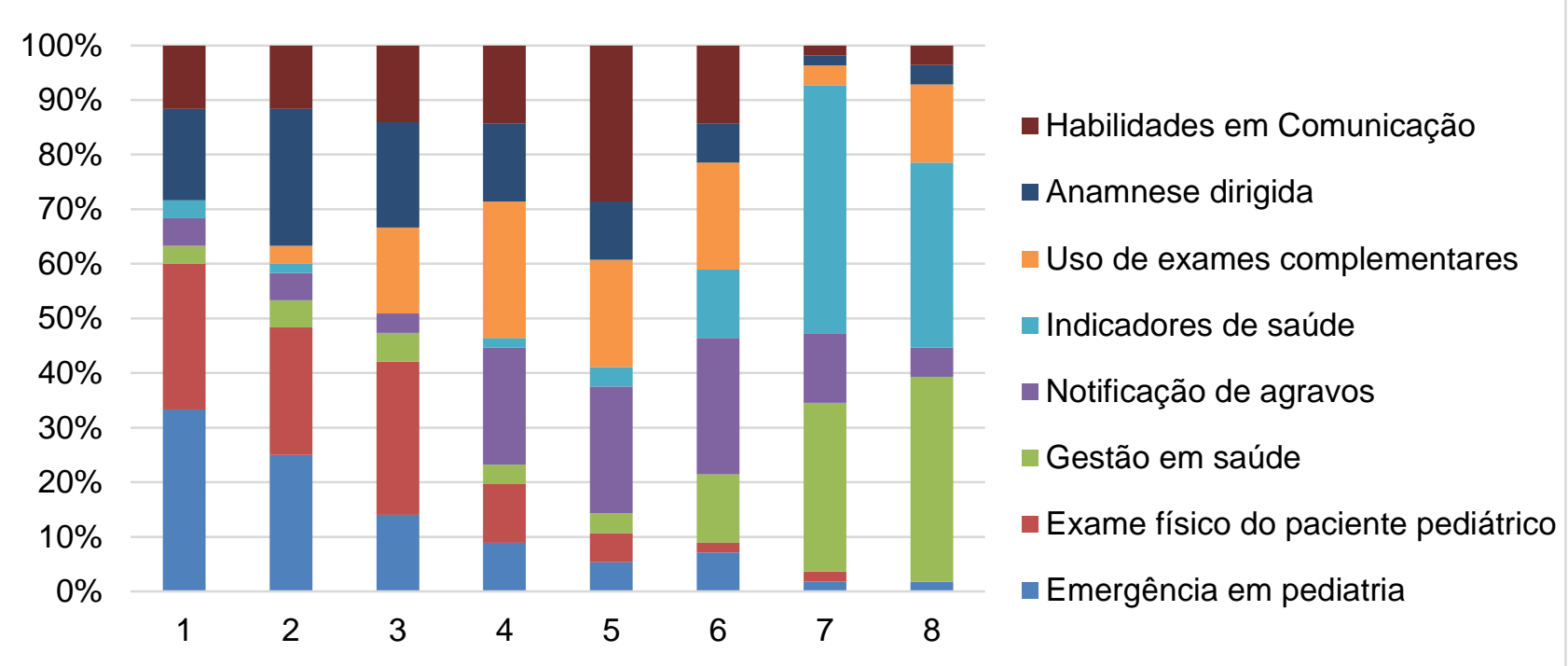

Fonte: Vaccarezza GF, et al., 2022.

\section{DISCUSSÃO}

Esse estudo teve como objetivo avaliar a percepção dos alunos e suas emoções em relação a uma atividade de simulação em gestão em saúde. Nos resultados demonstraram uma ótima aceitação dos alunos a simulação, conforme achados de estudos anteriores utilizando a simulação (WARREN JN, et al., 2016). 
Diferentemente dos outros estudos, a implementação do ensino baseado em simulação na área de gestão em saúde é rara, sendo que não encontramos nenhuma publicação que pudesse nos guiar. Interessante que os alunos relataram diversas emoções negativas vivenciadas por causa desta experiência. Tais emoções foram discutidas em um ambiente seguro na qual possibilitou que o aluno tivesse uma experiência prévia a prática profissional. Estas emoções podem dificultar 0 ato profissional principalmente por se tratar de emoções de raiva e indignação. Além disso, a prolongação dessas emoções negativas pode levar a um aumento de sintomas depressivos entre os estudantes (FOOKS P e SNUDDEN CM, 2016; MELO-CARRILLO A, et al., 2012; COSTA EFO, et al., 2012).

Neste contexto, mais uma vez vale a reflexão de importância da capacitação docente para lidar de forma satisfatória com os estudantes e desta forma atingir o resultado esperado com retenção do conhecimento adequada. É interessante como a percepção discente está sempre mais atrelada a assistência técnica, mesmo em cenários onde a condução assistencial e administrativa pode significar o bem-estar do paciente em outras dimensões. Para superar as barreiras ao desenvolvimento e permitir um treinamento de uma liderança eficaz e o treinamento em habilidades de gestão em saúde, esforços são necessários para trazer desenvolvimento a gestão para todos os estudantes de medicina desde o início de sua formação (MYERS CG e PRONOVOST PJ, 2017).

Infelizmente, essas temáticas, embora sejam conhecidas pelos discentes, são ainda trabalhadas de forma conteudistas ou apenas na observação assistencial direta, que nem sempre são acompanhadas de reflexão posterior ao caso. Existem discussões globais sobre como alinhar a educação médica com as mudanças socioeconômicas e as necessidades do sistema de saúde são recorrentes (HARTLEY K, 2016).

É mandatório que estratégias ativas de aprendizagem sejam incorporadas nos currículos médicos brasileiros de forma consistente e longitudinal, especialmente as que permitem o vivenciar de situações reais. Discutir gestão de forma aprofundada é visto com grande entusiasmo pelos coordenadores acadêmicos, porém o ensino ainda se dá de forma quase que exclusivamente de forma teórica. Apesar do Brasil ter aumentado a capacidade de formar médicos especialistas cerca de $40 \%$ dos médicos brasileiros não são especialistas e acabam atuando na atenção primária e serviços de urgência e emergência. Sendo assim, desenvolver habilidades para gerenciar conflitos e conhecer o sistema de saúde para encaminhamento de situações de risco é uma capacidade desejável na formação do profissional médico que exerce constantemente papéis de liderança (SCHEFFER M, et al., 2020).

O fato é que a realidade sanitária atual exige um esforço adicional no processo de capacitação dos recursos humanos, tendo em vista a relativamente escassa disponibilidade de profissionais com formação generalista e vocacionados às políticas públicas de saúde (ANDRADE JBC, et al., 2014; TEMPSKI P, et al., 2012). Propor acordos e gerenciar conflitos de forma equilibrada, ética e baseada em argumentos, promovem diferencial na rotina em qualquer gestão de saúde. O papel de liderança do médico tem sido cada vez reconhecido dentro da profissão como uma competência a ser desenvolvida, e que isso deve começar com mais oportunidades formais ao longo da graduação (FOOKS P e SNUDDEN CM, 2016).

As competências de liderança e gestão precisam ser treinadas e desenvolvidas, sem diminuir a importância das outras competências relacionadas a atenção à saúde ou demais conteúdos, assim como o conceito de liderança pode sobrepor dois termos semelhantes, gestão e administração. Alguns pesquisadores distinguem entre liderança, administração e gestão. Independente da etimologia, todas as três dimensões são identificadas como funções críticas da atividade organizacional (FOOKS P e SNUDDEN CM, 2016).

\section{CONCLUSÃO}

A formação médica brasileira em gestão em saúde é um aspecto essencial da formação profissional, sendo necessário a criação de estratégias educacionais que de fato levem a retenção deste conhecimento e suas competências. Esse estudo demonstrou que apesar da falta de literatura nesta temática, é possível sua inclusão curricular associada ao estágio na atenção primária em saúde. A vivência baseada pela simulação, promove excelentes taxas de retenção do conhecimento e, neste artigo foi descrito a excelente aceitação do método e compreensão das temáticas pelos discentes. 


\section{REFERÊNCIAS}

1. ANDRADE JBC, et al. Contexto de formação e sofrimento psíquico de estudantes de medicina. Revista Brasileira de Educação Médica, 2014; 38: 231-242.

2. BRANDÃO CS, et al. Centros de simulação e projeto pedagógico: dois lados da mesma moeda. Scientia Medica, 2018; 28; 1-10.

3. BRANDÃO CFS, FERNANDES DC. Importance and challenges of simulation training in healthcare. Scientia Medica, 2018; 28(1): 1.

4. BRASIL. Portaria Interministerial no 1.124, de 04 de agosto de 2015. Institui as diretrizes para a celebração dos Contratos Organizativos de Ação Pública Ensino-Saúde (COAPES) para o fortalecimento da Integração Ensino, Serviços e Comunidade no âmbito do SUS. Diário Oficial da União 2015.

5. BURSZTYN I. Diretrizes curriculares nacionais de 2014: um novo lugar para a Saúde Coletiva. Cadernos ABEM, 2015; 11: 7-19.

6. CHIAVERINI DH, et al. Guia prático de matriciamento em saúde mental. 2011.

7. COOK DA, et al. Technology-enhanced simulation for health professions education: a systematic review and metaanalysis. Jama, 2011; 306(9): 978-988.

8. COSTA EFO, et al. Sintomas depressivos entre internos de medicina em uma universidade pública brasileira. Revista da Associação Médica Brasileira,2012; 58: 53-59.

9. FOOKS P, SNUDDEN CM. Leadership and management: A neglected area of medical education. Medical Teacher, 2016; 38(10): 1072-1073.

10. FREITAS RJM, et al. Violência contra crianças/adolescentes em sofrimento psíquico e cuidado de enfermagem: reflexões da fenomenologia social. Revista Gaúcha de Enfermagem, 2016; 37.

11. GABA DM. The future vision of simulation in healthcare. Simulation in Healthcare, 2007; 2(2): $126-135$.

12. HARTLEY K. Untangling approaches to management and leadership across systems of medical education. BMC Health Services Research, 2016; 16(2): 33-43.

13. KUSSAKAWA DHB, ANTONIO CA. Os eixos estruturantes das diretrizes curriculares nacionais dos cursos de Medicina no Brasil. Revista Docência do Ensino Superior, 2017; 7(1): 165-184.

14. MYERS CG, PRONOVOST PJ. Making Management Skills a Core Component of Medical Education. Academic Medicine, 2017; 92(5): 582-584.

15. MCGAGHIE WC, et al. A critical review of simulation-based medical education research: 2003-2009. Medical Education, 2010; 44(1): 50-63.

16. MELO-CARRILLO A, et al. Depressive symptoms among Mexican medical students: High prevalence and the effect of a group psychoeducation intervention. Journal of Affective Disorders, 2012; 136(3): 1098-1103.

17. MINISTÉRIO DA EDUCAÇÃO. Conselho Nacional de Educação. Câmara de Educação Superior. Resolução CNE/ CES no 3, de 20 de junho de 2014. Diretrizes Curriculares Nacionais do Curso de Graduação em Medicina. Brasília; 2014.

18. MOTOLA I, et al. Simulation in healthcare education: a best evidence practical guide. AMEE Guide No. 82 . Medical Teacher, 2013; 35(10): e1511-e1530.

19. RODRIGUES NCP, et al. The increase in domestic violence in Brazil from 2009-2014. Ciência \& Saúde Coletiva, $2017 ; 22: 2873-2880$.

20. SCHEFFER M. et al. Demografia médica no Brasil 2020. São Paulo: FMUSP, CFM, 2020.

21. TEMPSKI $P$, et al. What do medical students think about their quality of life? A qualitative study. BMC Medical Education, 2012; 12(1): 1-8.

22. WARREN JN, et al. A systematic review of the effectiveness of simulation-based education on satisfaction and learning outcomes in nurse practitioner programs. Nurse Education Today, 2016; 46: 99-108. 